\title{
The Modular Structure of Kauffman Networks
}

\author{
U. Bastolla ${ }^{1,2}$ and G. Parisi ${ }^{1}$
}

October 2, 2018

\author{
${ }^{1}$ Dipartimento di Fisica, Università "La Sapienza", P.le Aldo Moro 2, I-00185 Roma Italy \\ ${ }^{2}$ HLRZ, Forschungszentrum Jülich, D-52425 Jülich Germany \\ Keywords: Disordered Systems, Genetic Regulatory Networks, \\ Random Boolean Networks, Cellular Automata
}

\begin{abstract}
This is the second paper of a series of two about the structural properties that influence the asymptotic dynamics of Random Boolean Networks. Here we study the functionally independent clusters in which the relevant elements, introduced and studied in our first paper [3], are subdivided. We show that the phase transition in Random Boolean Networks can also be described as a percolation transition. The statistical properties of the clusters of relevant elements (that we call modules) give an insight on the scaling behavior of the attractors of the critical networks that, according to Kauffman, have a biological analogy as a model of genetic regulatory systems.
\end{abstract}

\section{Introduction}

Kauffman networks are networks of randomly interconnected binary elements under reciprocal regulation. Every node is controlled by $K$ elements extracted at random in the set of the nodes. The boolean control rules, which associate a binary value to every configuration of the controlling elements, are also extracted at random at the beginning and kept fixed during the evolution of the system.

The relevant parameter of the distribution of the control rules is $\rho$, the probability that two different configurations of the controlling elements produce a different output. For every value of the bare connectivity $K$ there is a critical value of $\rho, \rho_{c}=1 / K$, which separates a frozen phase (small $\rho$ ) from a chaotic one (large $\rho$ ). According to Kauffman [1], this model reproduces on its critical line some features of the genetic regulatory systems acting in the cells.

In the preceding paper [3] we defined the relevant elements of Kauffman networks, which are the only elements that influence the asymptotic dynamics, and we measured their distribution. We refer to that paper for the description of the properties of the model, of the distribution of the relevant elements and of the relations between the number of relevant elements and the properties of the limit cycles of the dynamics. In the present paper 
we are going to describe how such elements are spontaneously organized into dynamically disconnected clusters that we will call modules.

It is known [7] that some of the elements in the network evolve to a constant state independent of the initial configuration and don't take part to the asymptotic dynamics. The relevant elements are the elements whose state is not constant and that control at least one relevant element. Thus every relevant element must be controlled by at least one relevant element (eventually itself) and must control at least one of them (eventually itself). It is then clear that loops of relevant elements must exist.

The set of relevant elements can thus be partitioned into different clusters, that we call modules, defined by the following equivalence relation: two relevant elements belong to the same module if one of them controls the other one. It is clear from this definition that elements in different modules cannot communicate among each other after a transient time: thus the dynamics induce spontaneously upon the network a clusterization of the relevant elements. The number of the limit cycles and their lengths depend only on this modular organization.

As expected, the statistical properties of the modules are very different in the two dynamical regimes of the model.

- In the frozen phase the number of relevant elements remains finite in the infinite size limit [ [G, 3]. In this case, on the ground of the argument presented in [3], we expect that the modules are, in the infinite size limit, loops of effective connectivity exactly equal to 1 (i.e., every relevant element is under the control of only one relevant element), and that the distribution of modules and, consequently, the number and the length of the attractors depend in this phase only on the parameter $c=K \rho<1$.

- In the chaotic phase the number of relevant elements is proportional to $N$, and the number of irrelevant elements tends to zero very fast as the bare connectivity increases (already for $K=3$ and $\rho=1 / 2$ the fraction of irrelevant elements is about 0.01 for an infinite system). The set of the relevant elements is fully connected in the infinite size limit, where the number of modules tends to 1 also for $K=3$ and $\rho=1 / 2$, as we shall see below. Nevertheless, in chaotic systems with low connectivity some signatures of a modular organization are present as a finite size effect.

- At the border between the two phases, the critical line shows an interesting behavior. Here the number of relevant elements scales as $\sqrt{N}$, i.e. the number of relevant elements increases with system size but they become more and more sparse. In this situation we expect that also the number of modules grows with system size, and that is actually what we observe in our simulations. The increase of the average number of modules is slow, apparently logarithmic in $N$, but it is responsible of important effects, such as the fact that the average weights of attraction basins tends to zero in the infinite size limit, or that the distributions of the length and the number of the limit cycles become broader and broader as $N$ increases.

Thus the phase transition in Kauffman model is reminiscent of a percolation transition. Below the threshold, the set of relevant elements is divided into a finite number of finite clusters. Above, it is completely connected. In critical systems, an infinite number of 
clusters is present. In this case it is an open problem whether the probability that all the relevant elements are connected tends to zero or to a finite limit in the infinite size limit.

Derrida and Stauffer were the first ones to notice the analogy with percolation theory for a lattice version of Kauffman model [5]. The study of the modular organization shows that the analogy with percolation theory remains pertinent also in the model with random neighbors.

We start presenting our numerical results from the statistical analysis of the divisors of cycle lengths (section 2), which allows the simplest numerical investigation of the modular organization. Section 3 is about the direct measure of the distribution of the number and size of modules, both in the chaotic phase and on the critical line. In section 4 we show how the number of modules and the effective connectivity depend on the number of relevant elements. A simple argument predicts the qualitative behavior of these quantities in agreement with numerical results. Section 5 concerns the relation between the number of modules and the length and number of the limit cycles, and section 6 deals with the distribution of attraction basins weights, interpreting results that we presented in [8]. An overall discussion concludes the paper.

\section{Divisors of cycle length}

\subsection{Analytic considerations in the frozen phase}

The distributions of the length of the cycles in critical networks $(K=2, \rho=1 / 2)$ and supercritical networks close to the phase transition $(K=3, \rho=1 / 2)$ show an oscillatory behavior: the cycles of even length are more frequent than the odd ones (in other words, the closing probability is larger when a cycle of even length is reached) [6]. Figure [1 shows the period distribution for a network on the critical line, with $K=4$ and $\rho=1 / 4$.

This is what should be expected if the relevant elements are subdivided into independent modules. In this case an attractor is the composition of the local cycles of the different modules, and its length is the least common multiple of the lengths of the local cycles, so that if at least one of the modules has a local cycle of even length also the global cycle will be even. Studying in more detail the statistical properties of the numbers that are integer divisors of cycle length it is thus possible to test this hypothesis and to obtain qualitative informations about the distribution of the modules, while the direct inspection of the modules is numerically more cumbersome (with this method it is enough to simulate one or two trajectories in every network, while for the direct study of the relevant elements and of the modules several hundreds of trajectories must be simulated).

We simulated two large systems in the chaotic phase and on the critical line, generating thousands of sample networks and two trajectories on each of them. For any small integer $l$ up to $l_{\max }$ we measured the probability to find an attractor with length multiple of $l, P_{d}(l)$, and, only for those networks where the two trajectories had reached different attractors, we measured the conditional probability that the length of the second cycle is multiple of $l$ given that the length of the first one is multiple of $l$ (we call this quantity $P_{d}(l \mid l)$ ). We measured such quantities for different sets of networks, defined by the condition that both cycles are shorter than a threshold length $L_{0}$, and studied how the results vary as a function of $L_{0}$. 


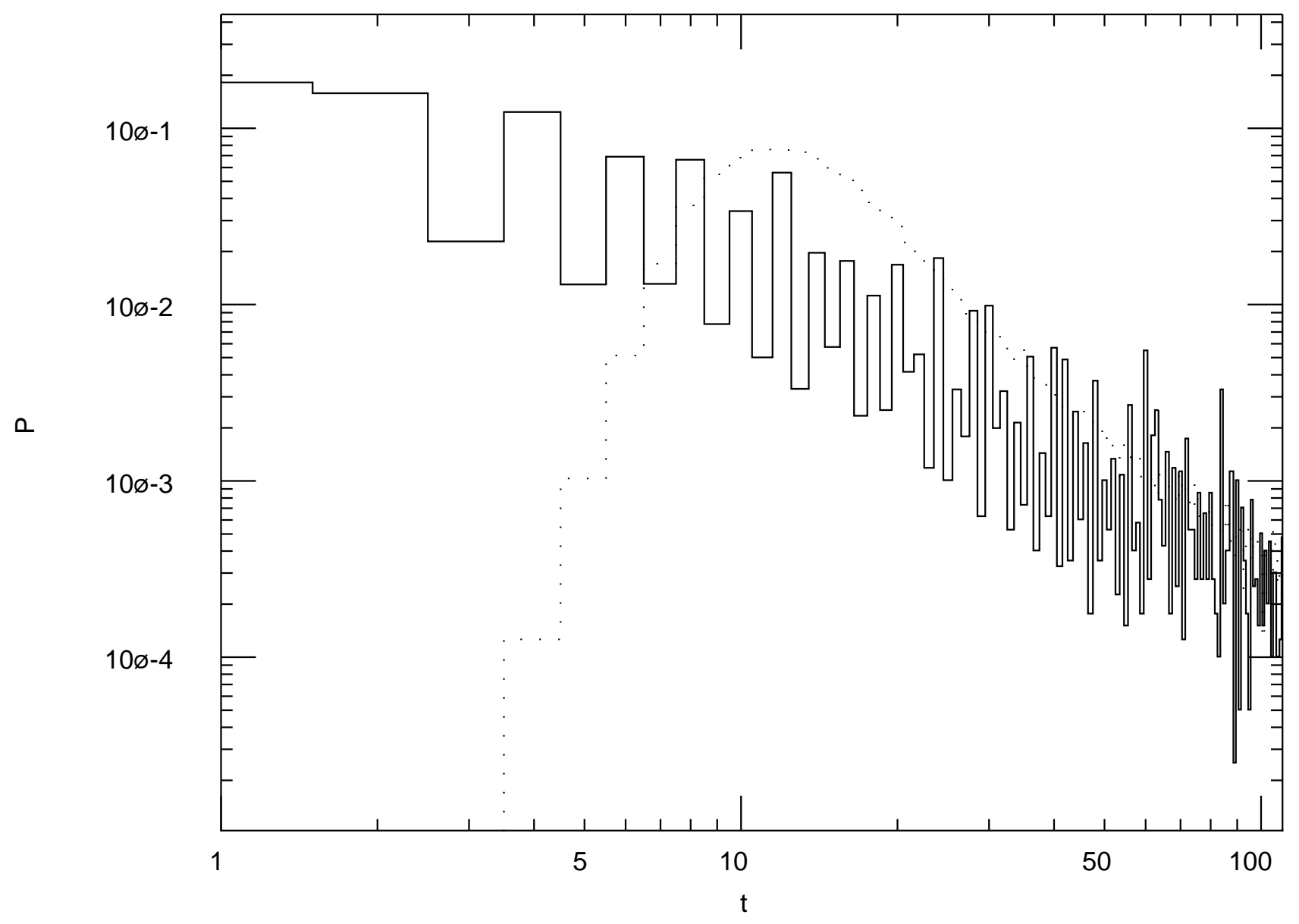

Figure 1: Probability to find a cycle with period $t$ in a random network with $K=2, \rho=1 / 2$ and $N=120$. The dotted line is the probability that the transient time is equal to $t$. 
As discussed in [3], there is a positive correlation between the length of the cycles and the number of relevant elements in a network, so that we expect that the condition imposed on the length of the cycles selects networks with less and less relevant elements as $L_{0}$ decreases.

\subsection{Critical system}

We discuss separately the results obtained for the critical and for the chaotic system, starting from the former one, which is a system with 2160 elements and parameters $K=4$ and $\rho=\rho_{c}=1 / 4$.

In this case the modular organization is very evident. In the case of a Random Map, $P_{d}(l)$ is proportional to $1 / l$. In the present case, instead, we have to distinguish between values of $l$ which are prime numbers and values which are not prime. For $l$ prime the probability $P_{d}(l)$ has initially a value larger than $1 / l$ (for instance, $P_{d}(2)=0.87$ when cycles shorter than 512 are selected), but then decreases faster than $l^{-1}$. The decrease become faster when shorter cycles are selected. This fact is consistent with the hypothesis of the modular organization, and, within this interpretation, shows that the probability to find a module with local cycle larger than $l$ decays faster than $1 / l$, and even faster if the number of relevant elements is small.

If $l$ is not prime, on the other hand, $P_{d}(l)$ is larger than for the prime numbers nearby, and it is usually close to the product of the probability of its prime factors (see figure 2). This is a strong hint to interpret these prime factors as the length of the local cycles of individual modules

The effect of changing the threshold on cycle length, $L_{0}$, is shown in figure 3. For a fixed $l$, the probability to find a cycle whose length is a multiple of $l$ increases, reach a maximum value and then decreases. Since the abscissa can be related to an increasing number of relevant elements, a possible interpretation of this behavior is that the number of modules first increases with the number of relevant elements, then reaches a maximum value and ultimately decreases. We will see in section 4 that this is just what happens.

At last, the condition that we already found in the same network a cycle multiple of $l$ largely improves the probability to find another cycle multiple of $l$, consistently with the modular interpretation. This probability decreases when we select networks characterized by longer attractors (this can be interpreted as another indication of the fact that the number of modules may decrease as the number of relevant elements increases).

\subsection{Chaotic system}

The network that we examined is close to the critical phase: $K=3, \rho=1 / 2, N=100$. This is a very large size concerning the duration of the simulation, since in the chaotic phase the length of the cycles increases exponentially with system size, but it still shows important finite size effects. We think that the signs of the modular organization that we observed can disappear in an infinite system, since this signs decrease when we select networks with longer attractors, i.e. with more relevant elements, and in an infinite system the fraction of relevant elements tends to 0.99. A direct study of the modules for smaller systems shows in fact that the average number of modules tends to 1 in this limit (see next section). 


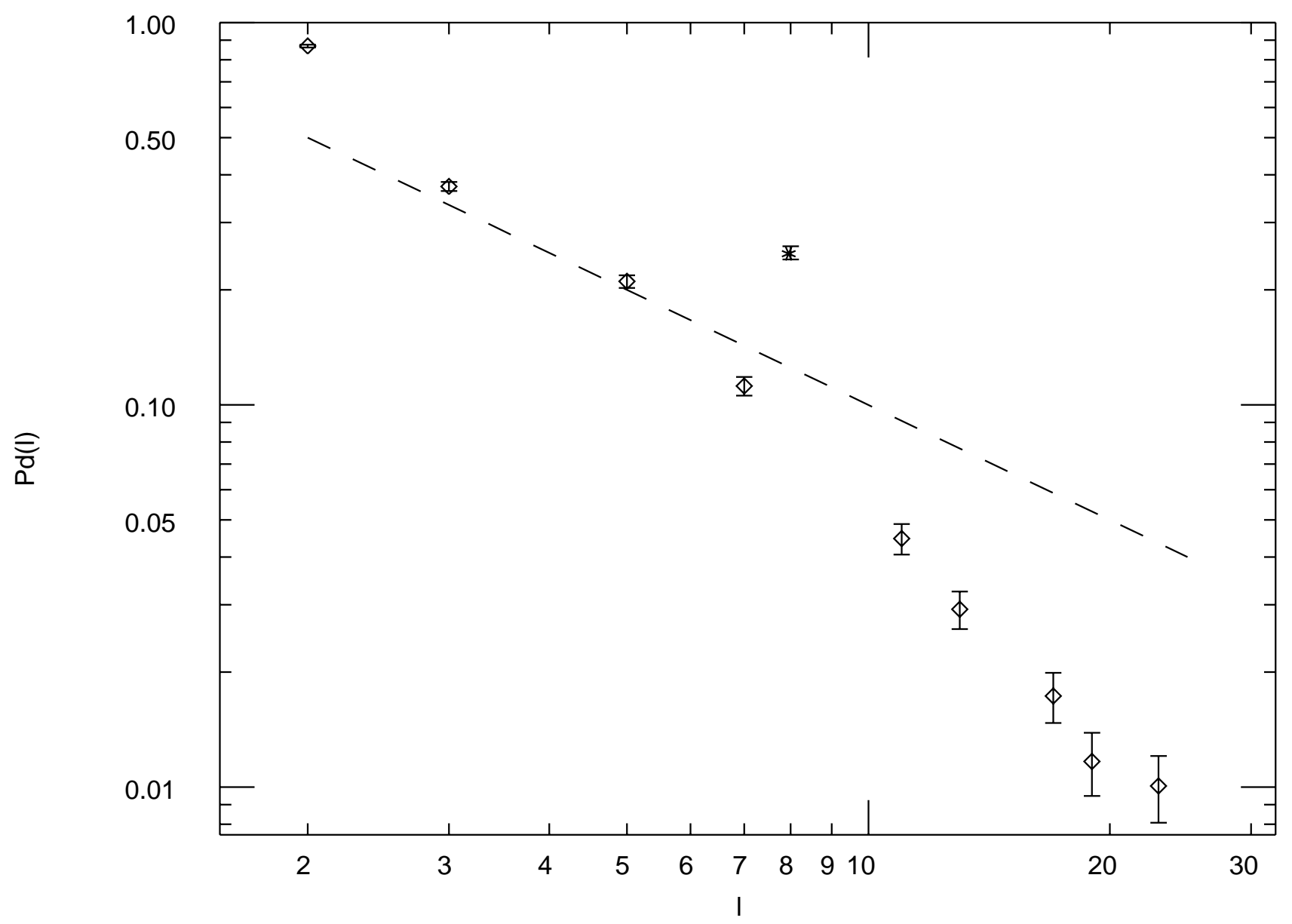

Figure 2: Probability to find a cycle of length multiple of $l$, where $l$ is a prime number, in critical networks with $K=4, \rho=1 / 4$ and $N=2160$. The dashed line represents $1 / l$. The isolated point above that line shows the same probability for $l=8$. 


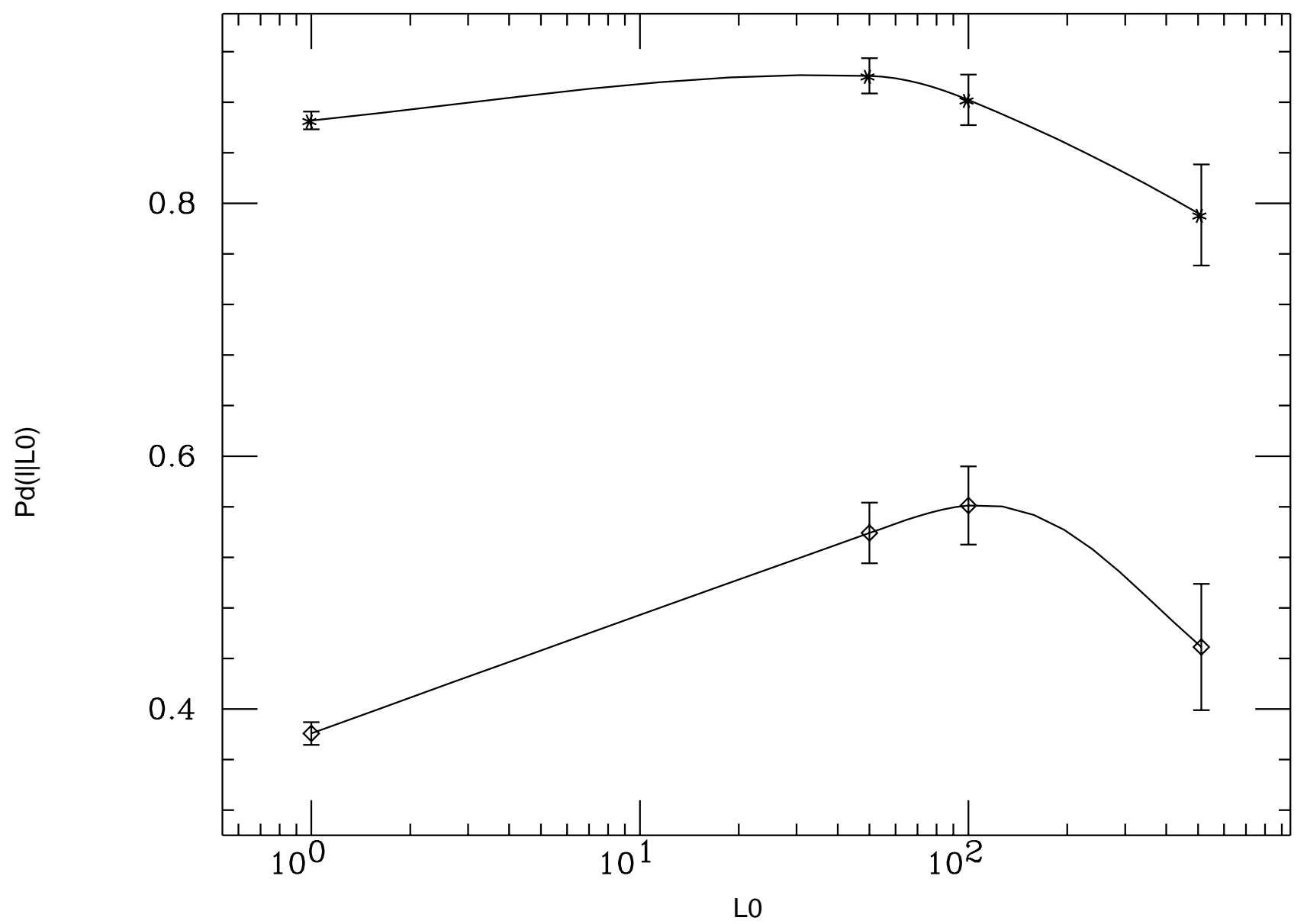

Figure 3: Probability to find a cycle of length multiple of $2\left(^{*}\right)$ and $3(\diamond)$ restricted to the set of cycles shorter than $L_{0}$, as a function of $L_{0}$, in critical networks with $K=4, \rho=1 / 4$ and $N=2160$. 
Also in this case, however, the probability to find a global period which is multiple of $l$ decreases faster than $1 / l$ when $l$ is a prime number, but the difference from $1 / l$ is smaller than in the previous case (in networks with attractors shorter than 50 steps, its value is 0.70 for $l=2$ and 0.29 for $l=3$ ). Also this probability is larger when $l$ may be decomposed into prime factors. However this features tend to disappear when larger cycles are selected, and $P_{d}(l)$ approaches $1 / l$ when the threshold $L_{0}$ increases, thus indicating that the modular organization tends to disappear when the number of relevant elements increases.

Correlations between the divisors of different cycles on the same network seem to be present (the conditional probability $P_{d}(l \mid l)$ is larger than $P_{d}(l)$ ), but they are of little significance (the difference between the two probabilities is, at most, of the order of two or three standard deviations) and our simulations can not even prove that such correlations exist. In any case the correlations, if any, are much smaller than in the critical system, and they appear to decrease when networks with longer attractors are selected.

\section{Distribution of modules}

In this section we deal with a direct study of the number of modules. Before presenting the results that we obtained, however, we shall describe briefly our algorithm, also in order to give an operative definition of the modules.

The first step, as it is discussed in [3], consists in identifying the stable elements. To this aim we simulate 300 trajectories for each of the networks that we generate. The stable elements are those ones that reach the same stable state in all the different runs. The set of the unstable elements is then reduced to the relevant elements alone (the ones that control at least one relevant element), and in this process the irrelevant connections are eliminated (the connection between $a$ and $b$ is irrelevant if the response function does not depend on the state of $a$ when all the stable values of the stable elements have been substituted in it, i.e. if the function reduced to the unstable arguments alone is a constant function of the state of $a$ ). Now every relevant element receives a different label, and the following procedure is iterated: if either $a$ controls $b$ or $b$ controls $a$, and their labels are different, their labels are set equal to the smaller one, and all the labels equal to the one that is changed are set to the same value. We check that no label is changed more than once in an iteration. When no label changes anymore the iterations are interrupted. At this point to every different label corresponds a different module, and we count their number and their size.

\subsection{Chaotic systems}

We start to present results relative to the chaotic phase, where the existence of a modular organization appears only as a finite size effect. We investigated numerically systems not far from the critical line, with $K=3, \rho=1 / 2$ and $N$ comprised between 20 and 75 . For such systems, the probability to generate a network with more than one module tends to zero roughly as $1 / N$.

Comparing this result with the analysis of the divisors of cycle length (section 2) we see that the modular organization is not the only explanation for the high frequency of even

periods, which would be smaller if it were due only to the presence of different modules. It is 
possible however that some other structures generalizing the notion of module can give the same effect and are present with larger probability. For example, we can define in-going and outgoing submodules as sets of relevant elements that do not, respectively, receive from or send to the outside any relevant signals. The length of a local cycle is then multiple of the period of all the in-going submodules, and the situation described in the previous section may occur because of the submodules. Another structure that could play a role is that of cycle-depending modules. Globally stable elements are defined as the elements that reach the same fixed state independently on the initial configuration. The elements stable on a cycle are a subset of those, and are defined as the elements whose value does not change along the cycle. Thus the network can be decomposed into cycle-depending modules that do not communicate between each other when the system is on the attractor. Once again, the period of the attractor is the least common multiple of the periods of all cycles of the partial modules that compose it. Or it may be that the statistical properties of the divisors of cycle length do not depend on the presence of modules.

However, it is clear that the modular organization tends to disappear in the infinite size limit.

\subsection{Critical systems}

The distributions of the number of modules in critical systems with $K=4$ and $\rho=1 / 4$ have a maximum corresponding to a single module and then decrease roughly exponentially, but with a rate that becomes slower and slower as system size increases (figure đ). The probability to find a single module, or no module at all (this means that all the elements are stable) decreases with system size, while the probabilities to find 2 or more modules increases. It is not clear whether the probability of networks with only one module tends to zero or to a finite limit. The average number of modules increases with system size. T, and the best fit is logarithmic:

$$
\left\langle N_{\text {mod }}\right\rangle \approx 0.40+0.39 \log N
$$

We prefer the logarithmic fit to a power law with a small exponent, also compatible with our data, for two reasons: first, because the inclusion of the largest $N$ value modifies the fit parameters by 5 percent in the first case and by 10 percent in the second one, and second because at the "critical point" $K=1, \rho=1$ the analogy between the modules and the cycles of a Random Map shows that the average number of modules grows logarithmically with system size in this case [4]. This point is not a real critical point since it is not at the border between two phases (with $K=1$ only the frozen phase is possible), but still the analogy with it can give useful indications.

Most of the modules have only one element, and the distribution of the size of the modules decreases monotonically. The probability to find a module with 1,2 or 3 elements depends very little on the system size $N$. On the other hand, this probability increases with $N$ for modules of large size (figure 5), consistently with what we saw in [3]: the number of relevant elements, which is the sum of the sizes of all the modules, scales as $\sqrt{N}$ on the critical line. 


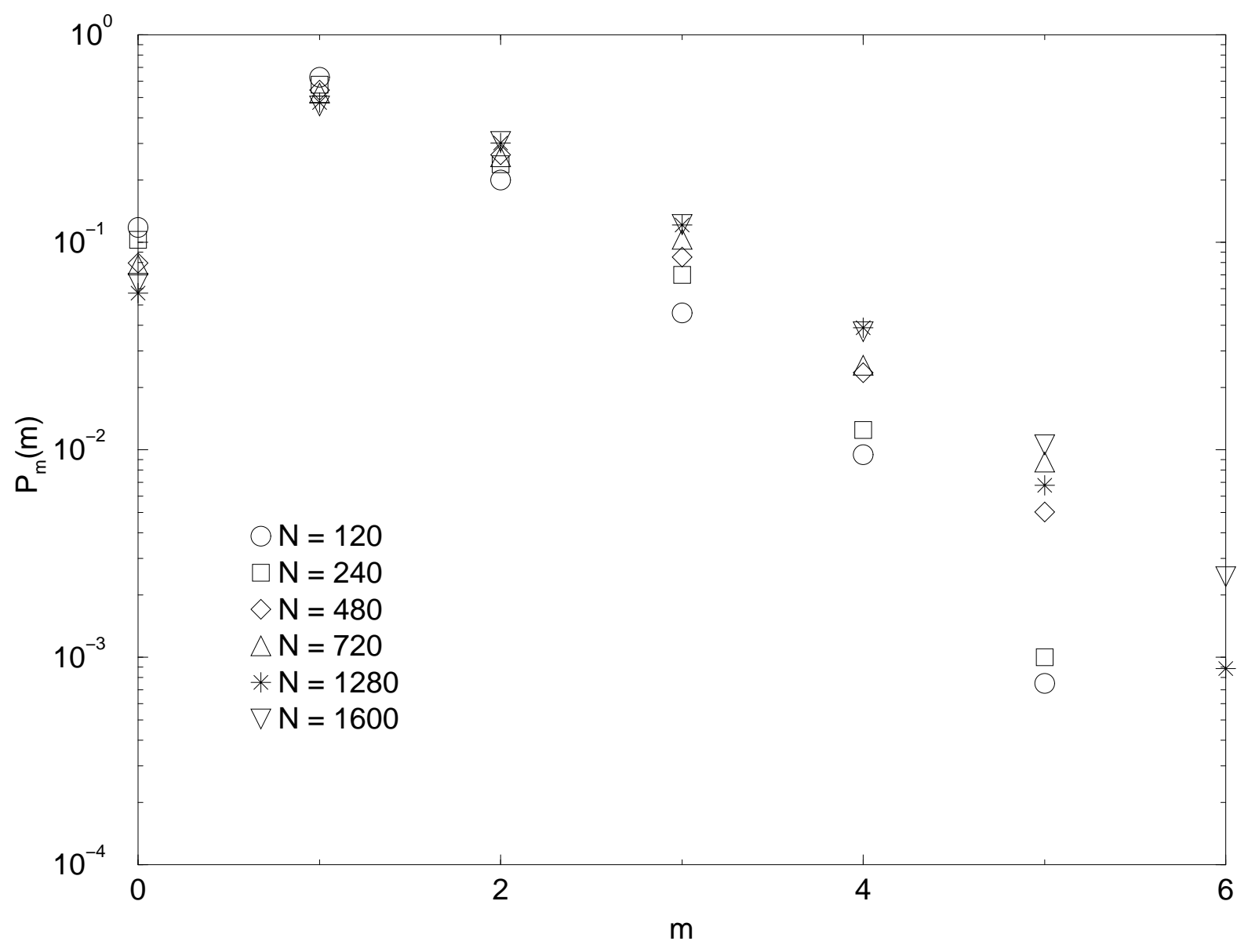

Figure 4: Distribution of the number of modules in a random network at the critical point $K=4$ and $\rho=1 / 4$. $N$ is ranging from 120 to 1600 . 


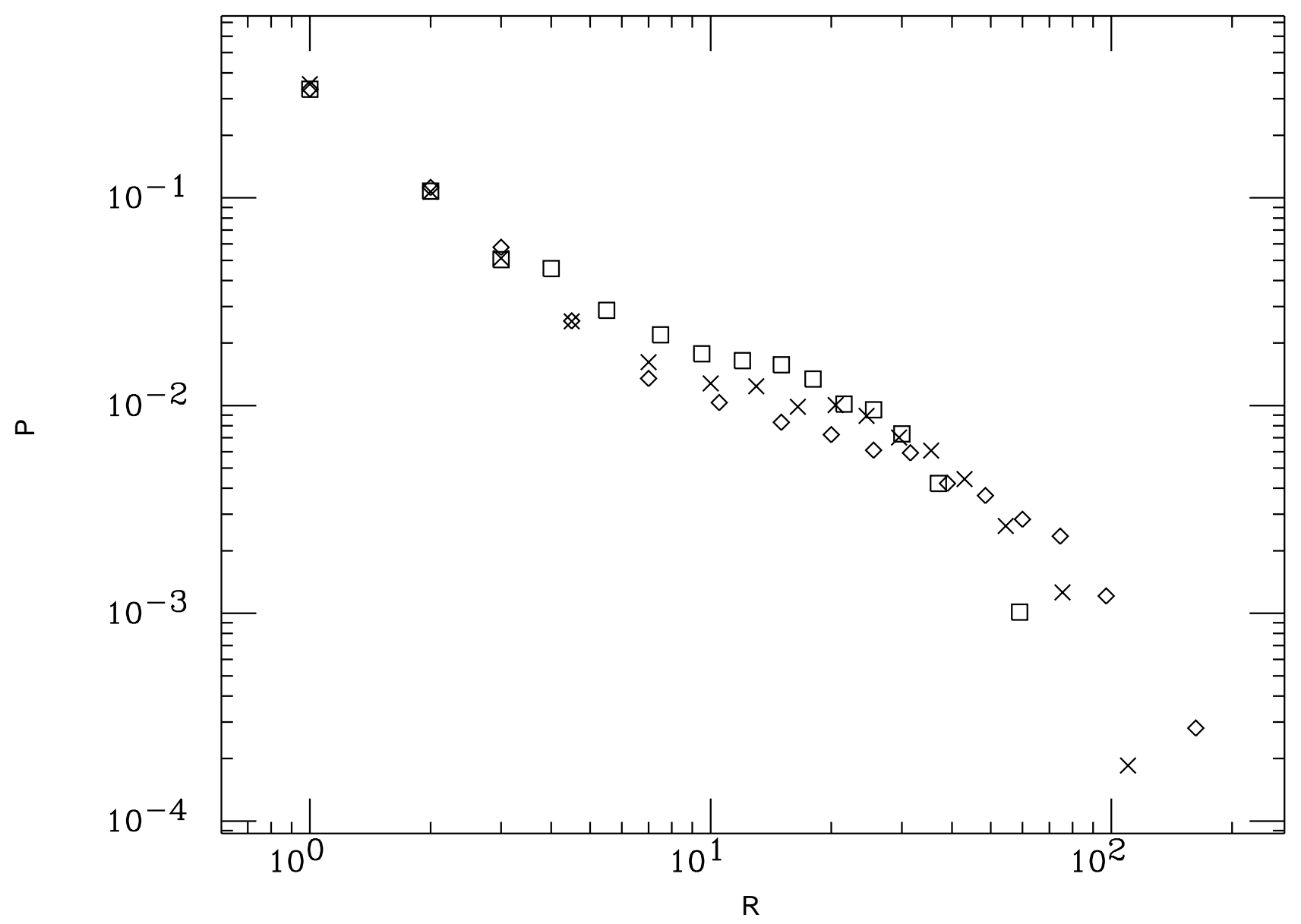

Figure 5: Distribution of the number of modules with $R$ relevant elements, at the critical point $K=4, \rho=1 / 4 ; N$ is respectively equal to 120 (squares), $240(X)$ and $480(\diamond)$. 


\section{Effective connectivity}

In this section we examine how the number of modules depends on the number of relevant elements. A useful notion to understand this relation is the effective connectivity of the network. Also in this case the simulations were done with critical networks with $K=4$ nd $\rho=1 / 4$.

We plot in fig. 6 the average number of modules as a function of the number of relevant elements in the network, $R$, for systems of different sizes. This quantity shows a non monotonic behavior: the number of modules increases with $R$ at the beginning, then it reaches a maximum and starts to decrease towards the value 1 .

The plot of the average number of relevant elements as a function of the number of modules (figure 7) contains a similar information. Contrarily to the naive expectation, the largest number of relevant elements is attained in networks with only one module. It seems that then the curve reaches a minimum and starts to increase, but we have the networks with such a large number of modules are very rare and we can not make any significant statement about this point.

It is not difficult to explain qualitatively this behavior. At this end we use the same argument that we used in [3] to estimate the scale of the number of relevant elements. We suppose to add a new element to a network with $R$ relevant element. If the new added element happens to be relevant, three situations are possible:

1. The new relevant element is added to a previously existing module and the number of modules does not change;

2. The new relevant element constitutes a new module itself and the number of modules increases by one unit;

3. The new relevant element connects two modules and the number of modules decreases by one unit.

When $N$ is large all other possibilities can be neglected. The situation is trivial in the frozen phase, where an asymptotic (for large $N$ ) distribution of the relevant elements is reached and all the three cases above have negligible probability, and in the chaotic phase, where only one module is present in systems of large size, but it is very interesting on the critical line, since the properties of the attractors vary according to which of the three situations is the typical one.

To understand how the number of modules depends on $R$ on the critical line, we have to compare case 2 and case 3. If, for a given value of $R$, the second case prevails, the number of modules increases with $R$. In the other case, it decreases. The probability of case 2 should not depend on $R$, while the probability of case 3 is proportional to $\sum_{i} n_{i} n_{j}$, where $n_{i}$ is the size of module $i$ and the sum runs over different modules. When $R$ increases, we should expect this quantity to increase so that case 3 prevails. The quantity that allows to understand quantitatively the situation is the average rescaled size of a module, defined as

$$
S_{2}=\left\langle\sum_{i} n_{i}^{2} /\left(\sum_{i} n_{i}\right)^{2}\right\rangle .
$$




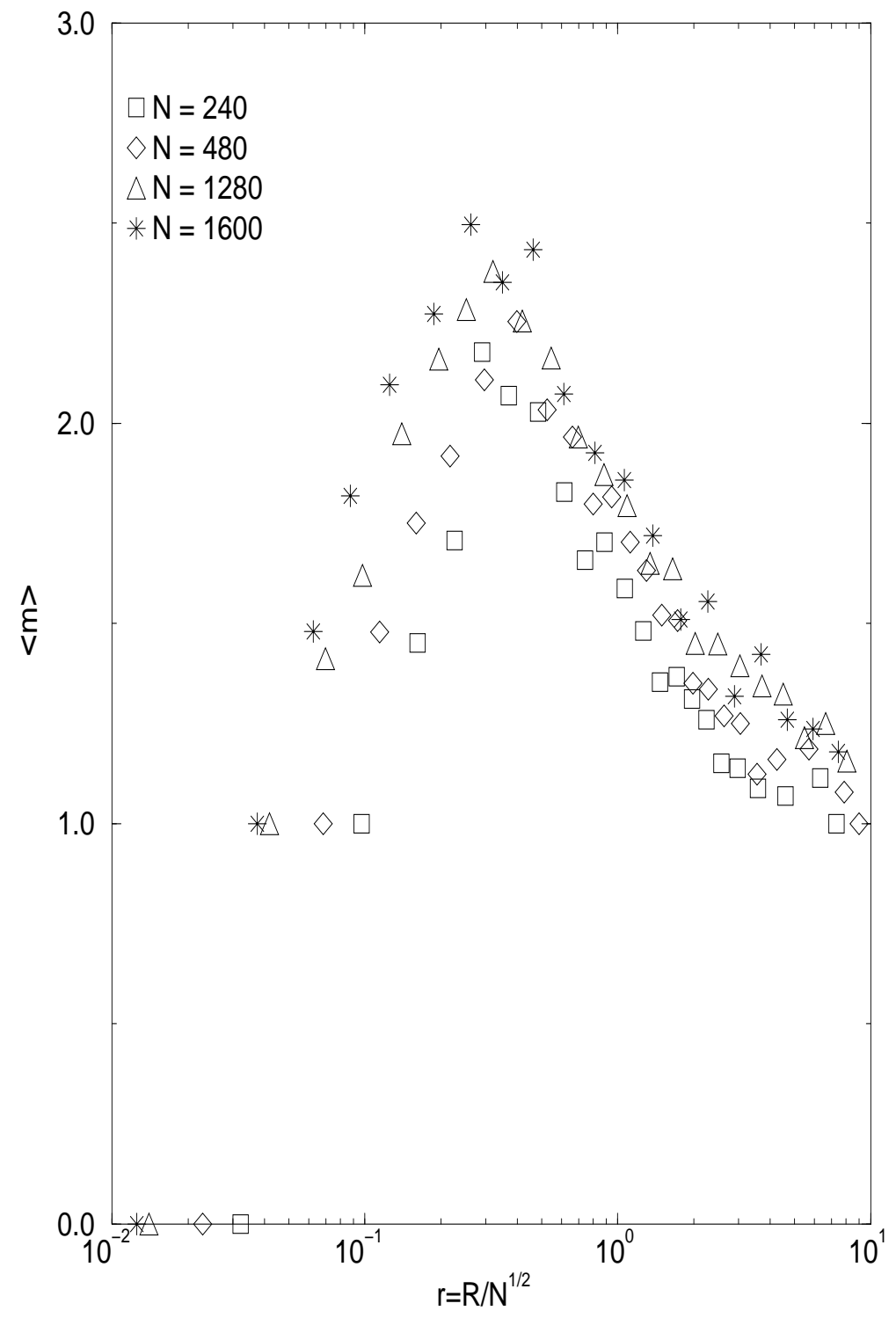

Figure 6: Average number of modules in networks with $R$ relevant nodes as a function of $r=R / N^{1 / 2}$ at the critical point $K=4, \rho=1 / 4$. 


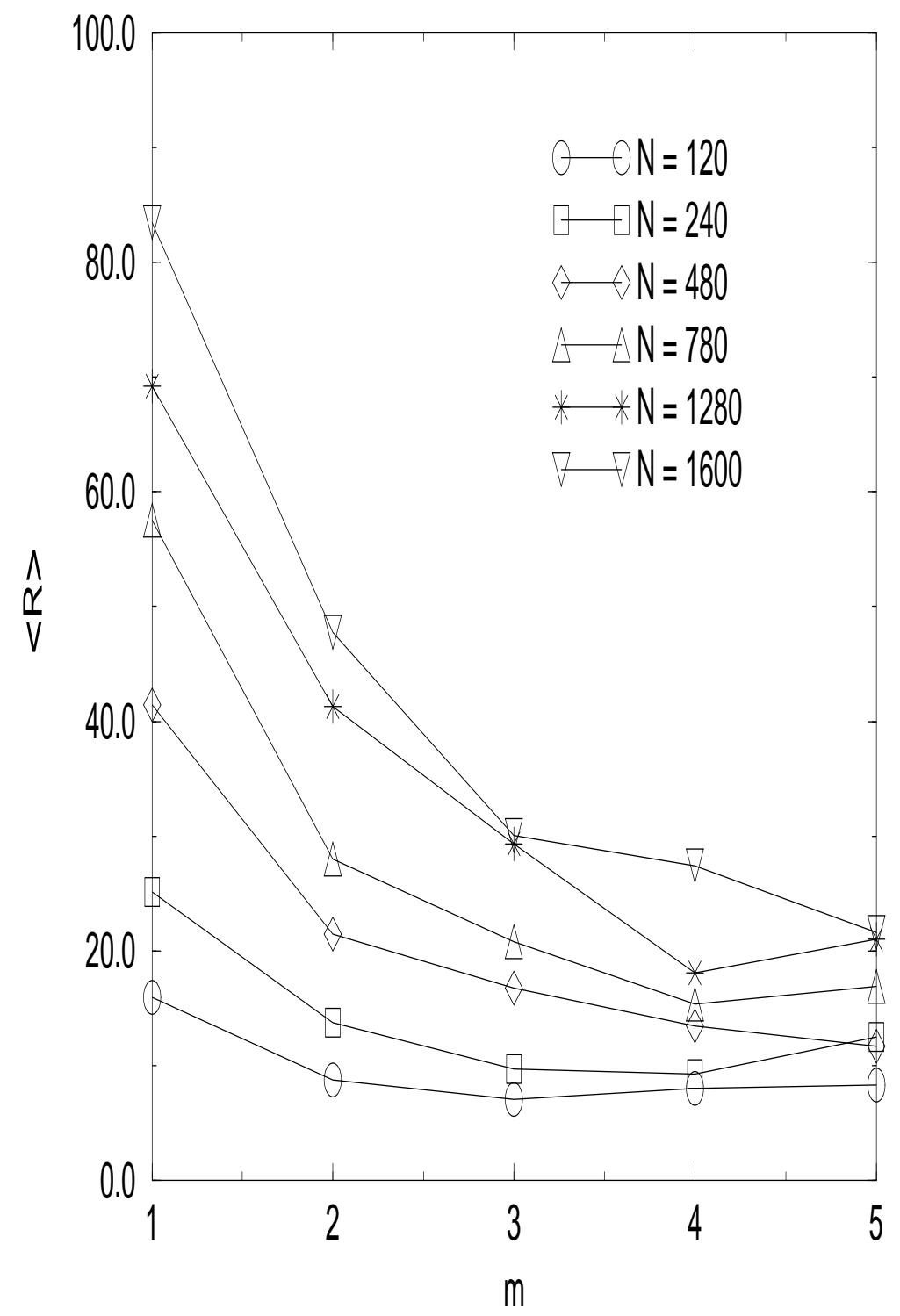

Figure 7: Average number of relevant elements in networks with $m$ modules at the critical point $K=4, \rho=1 / 4$. 
If this quantity is large (close to 1 ) case 2 prevails also for large values of $R=\sum_{i} n_{i}$, otherwise case 3 is the prevailing one.

This argument is supported by the study of the average number of relevant connections in the network, that we called the effective connectivity of the network. In critical networks, this quantity is much smaller than the bare connectivity, $K$, and tends to 1 as a power law when $N$ increases. We are interested to the behavior of $K_{\text {eff }}$ as a function of the number of relevant elements in the network (in other words, $K_{\text {eff }}(R)$ is a conditioned average, restricted to the set of networks with exactly $R$ relevant elements).

For large $R$ this function is asymptotic to a straight line with intercept close to 1 (see figure 8). It holds:

$$
K_{\text {eff }}(R) \approx a(N) R+b(N),
$$

where the parameter $a$ decreases as a power law: $a(N) \approx 1.7 N^{-0.93}$, and $b(N)$ tends to 1 from above (every relevant element must have at least one relevant connection, so that $K_{\text {eff }}$ can not be less than 1).

This is very close to what we expect on the basis of the above argument [3]. We expect that the conditional probability that the new element has two relevant connections, given that it is a relevant element, is proportional to $R / N^{2}$. This is a quantity of order $1 / N$, so this argument predicts $a(N) \propto 1 / N$, to be compared with our best numerical fit $a(N) \propto N^{-0.93}$. From this argument, we would expect also that $K_{\text {eff }}=b(N)+\langle R\rangle a(N)$ is equal to 1 plus a term vanishing as $1 / \sqrt{N}$. It turns out, however, that the correction decreases as $N^{-0.3}$ : this discrepancy is due to the term $b(N)$, and we don't have a good explanation for it.

The behavior of the effective connectivity and that of the number of modules as a function of $R$ show the existence of a crossover between two situations reminiscent of the two phases as the number of relevant elements changes along the critical line. For small $R$ we find networks with small effective connectivity: most of the modules are loops where each relevant element has only one connection, as it is typical in the frozen phase. To the opposite side, where $R$ is large, the networks are much more connected and there are few modules. This situation is similar to what happens in chaotic networks, but on the critical line only a finite number of elements have more than 1 connection in the infinite size limit.

We thus expect that the properties of the attractors change widely with the number of relevant elements, from a situation reminiscent of the frozen phase to a situation reminiscent of the chaotic phase. We dedicate the rest of this paper to describe how the properties of the attractors are influenced by the properties of the modules.

\section{$5 \quad$ Attractors and modules}

\footnotetext{
${ }^{1}$ In this context, we say that element $a$ has a connection with element $b$ only if $a$ is controlled by $b$, and not if $b$ is controlled by $a$.

${ }^{2}$ Of course, $K_{\text {eff }}$ should saturate to a value smaller than $K$ when all elements are relevant, but such networks occur with negligible probability on the critical line.
} 


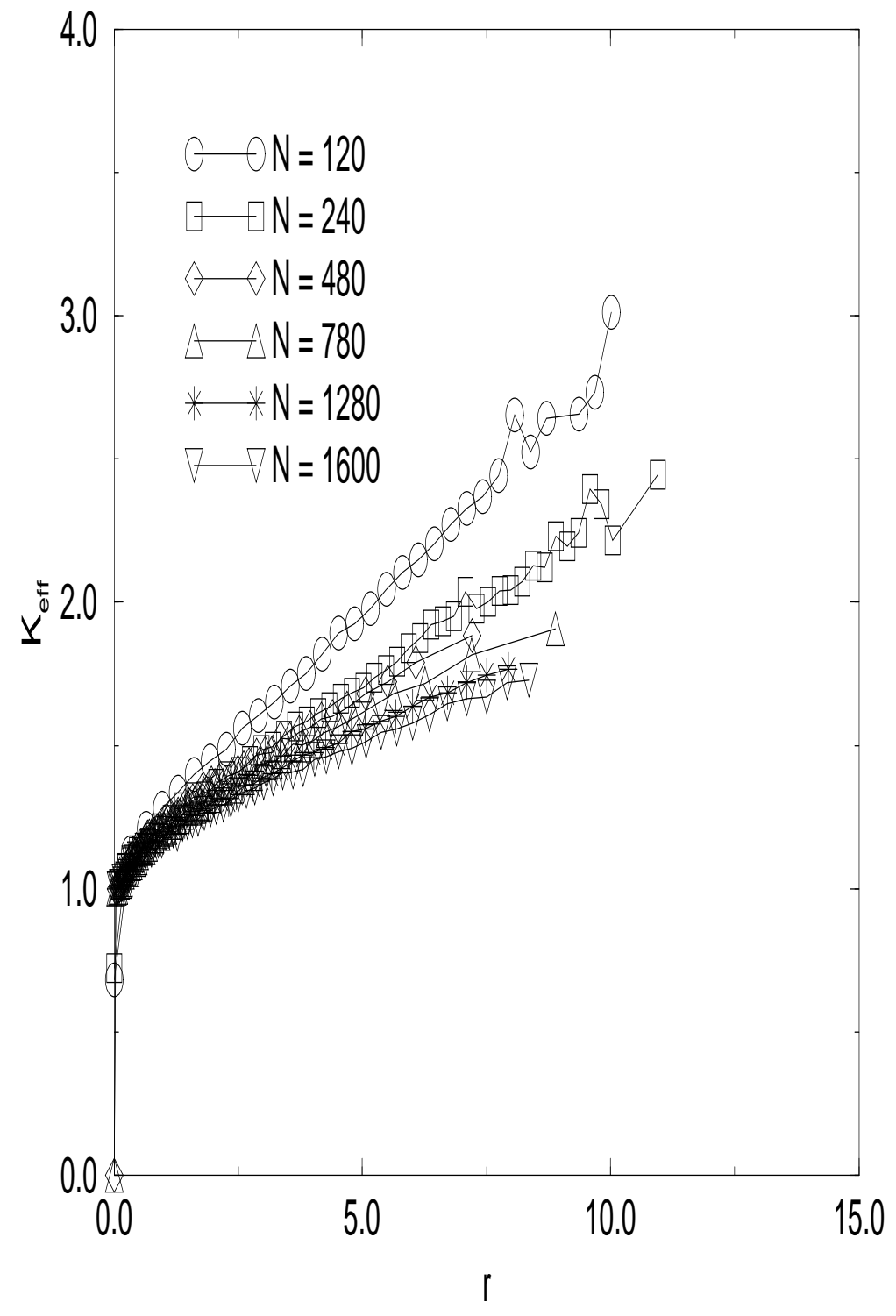

Figure 8: Effective connectivity in networks with $R$ relevant nodes as a function of $r=$ $R / N^{1 / 2}$ at the critical point $K=4, \rho=1 / 4$. 


\subsection{Simply connected modules}

We saw in the previous section that the effective connectivity of critical and frozen systems tends to 1 in the infinite size limit. This means that most of the elements receive and send only one relevant signal. Thus simply connected modules play an important role in critical networks, and are found with probability one in the frozen phase. Such modules were analytically studied by Flyvbjerg and Kjaer in the case of $K=1$ networks [ [⿴囗十 . Here we recall for completeness their properties, adding some more information.

A simply connected module is a loop of relevant elements $i=1, \cdots l$, such that the node $i-1$ controls node $i$ through the Boolean function $f_{i}(\sigma)$ (we assume periodic boundary conditions). There are only two kinds of non-constant Boolean functions of one Boolean argument: tautology $(f(\sigma)=\sigma)$ and contradiction $(f(\sigma)=\bar{\sigma})$.

The number and length of the cycles of such modules do not depend on the details of the distribution of the Boolean functions, but only on the parity of the number of negative functions [4]. All the $2^{l}$ configurations of the module belong to some cycle of length equal to an integer divisor of $l$ (if the number of negative functions is even) or of $2 l$ (if it is odd): in fact, after at most $l$ steps in the first case, and $2 l$ steps in the second one, every initial configuration is reproduced. This is the first important property: the length of the cycles increases only linearly with the size of the module.

The number $g_{l}(m)$ of cycles of length $m$ in a module of size $l$ is easily found. First, it is easy to see that $g_{l}(m)$ does not depend on $l$. In fact, let us assume an even number of negative functions. A module made out of $l$ elements may be thought of as the repetition of $k$ modules made of $m$ elements, as $m$ must be an integer divisor of $l=k m$. But for all the initial configurations whose periodicity is $m$ the dynamics is independent on the value of $k$; on the other hand, the configurations that are not periodic in space can not be reproduced after $m$ steps. For an odd number of negative functions the argument is exactly the same, but the $k$ modules of size $m$ can be equal only if $k$ is odd. Thus $g_{l}(m)$ depends only on $m$ and on the parity of the number of negative functions, that we shall denote by a superscript: (0) to indicate even, and (1) to indicate odd. It holds

$$
\begin{aligned}
\sum_{\{m \mid l / m \text { integer }\}} m g^{(0)}(m) & =2^{l}, \\
\sum_{\{m \mid l / m o d d\}} 2 m g^{(0)}(2 m) & =2^{l},
\end{aligned}
$$

whence it can be seen that $g^{(0)}(l)$ is asymptotic to $2^{l} / l$ and $g^{(1)}(2 l)$ is asymptotic to $2^{(l-1)} / l$. In the large $l$ limit, most of the cycles have the maximal length.

\subsection{Global attractors}

A global attractor is made out of the composition of the limit cycles of all the modules. Its period is thus the least common multiple of the periods of the cycles that compose it. The number of attractors is larger than or equal to the product of the number of cycles in the different modules. In fact the number of attractors that can be formed with a set of limit cycles, each one taken from a different module, is equal to the maximum common divisor of the periods of the cycles. 
Even in the absence of an analytic description of the statistical properties of the modules, the study of the modular structure allows us to better interpret the numerical results about the distributions of the number and of the length of the attractors in critical networks [B] and sheds light on the large size behavior of these quantities. In the following we will concentrate our discussion on critical networks.

First, we note that the two extremal situations of very high and very low connectivity, which are reminiscent respectively of the chaotic and of the frozen phase, are expected to be very different also from the point of view of the properties of the attractors.

In high connectivity critical networks the attractors are reminiscent of the chaotic phase (we have to remember, nevertheless, that for critical networks $K_{\text {eff }}$ tends to 1 in the infinite size limit, so that high connectivity means that there is a large but finite number of elements with two connections. Such networks are characterized, as it is discussed above, by few modules of large size). We observed in fact that the average length of the cycles increases exponentially with the number of relevant elements for large $R$, as it happens in the chaotic phase. Thus in such networks the average length of the cycles should increase as a stretched exponential of system size. This result is consistent with the fact that the distribution of the length of the cycles seems to have an effective scale increasing as a stretched exponential 8]. Similarly, we expect that the number of attractors in such networks is not very large (in the chaotic phase the number of attractors increases linearly with the number of relevant elements), but we don't have data to compare to this prediction.

In low connectivity critical networks there are several modules with very few elements. Most of the modules are just loops with effective connectivity exactly equal to 1 , so that the length of the local periods is proportional to the size of the loop and the number of cycles increases almost exponentially with it. Thus, in this situation we expect to find a very large number of attractors, exponentially increasing with the number of relevant elements. This means that in such networks the number of cycles should increase approximately as a stretched exponential of system size. The distribution of the number of attractors is consistent with this prediction, since it has an effective scale increasing as a stretched exponential of system size. On the other hand, we expect to find in such networks short cycles: the typical length should be the minimal common multiple of the size of the modules, and it should increase as the square root of the number of elements, as far as the number of modules is not very large. But, as $N$ increases, this scaling should be lost and substituted by a faster increase.

It can thus be understood why both the distributions of the length of the cycles and the distribution of their number become broader and broader when system size increases: in the ensemble of critical networks quite different situations coexist. It seems also that networks characterized by a small number of short attractors (meaning by this that the number and the length increase not faster than a power law of $N$ ) can be found only if the number of relevant elements is very small, thus with vanishing probability as $N$ increases. However, this qualitative picture still requires more accurate further investigations to be confirmed or rejected. 


\section{Attraction basins}

While the number and the length of the attractors depend only on the number and the size of the modules, this is not true for the size of the attraction basins.

In fact, when we extract at random an initial configuration, the elements that are going to become stable are not yet stable and the modules are not yet formed (the case $K=1$ is an exception, since in this case the modules are present from the beginning), since the elements of different modules can still communicate. Thus the attraction basins of two critical networks with the same modular structure but with different bare connectivity don't need to be identical. In fact, we expect that the attraction basins for critical systems are larger the larger is $K$, since then $\rho_{c}=1 / K$ is smaller and a large number of the input configurations produce the same state. In the following, we will be mainly dealing with critical systems, unless otherwise stated.

The simulations of critical systems with $K=2$ and $K=4$ confirm that, for a given system size, the attraction basins are on the average larger for $K=4$ than for $K=2$ [8], but show also that the main characteristics of the distribution of the weight of the attraction basins are the same in both cases. The weight of the attraction basin $\alpha, W_{\alpha}$, is defined as the fraction of configurations that evolve towards attractor $\alpha$. This quantity has to be averaged over all the attractors and over several realizations of the dynamical rules. Following [9] we use the notation

$$
\left\langle Y_{2}\right\rangle=\sum_{\alpha}\left\langle W_{\alpha}^{2}\right\rangle
$$

(the angular brackets represent the average over the disorder).

For both the $K$ values that we examined, $\left\langle Y_{2}\right\rangle$ vanishes as system size increases, and in both cases our data are compatible with a power law decay with the same exponent for the two parameters considered, suggesting that this behavior is universal along the critical line. Moments of higher order of the weight of the attraction basins also seem to vanish as a power law of system size, but with an exponent which is not proportional to the order of the moment, so that a typical weight of the attraction basins can not be defined and the distribution of the weights has multi-fractal properties [10].

The fact that the average weight of the attractor basins vanish in the infinite size limit can be qualitatively explained considering the modular structure of critical networks. We measured $\left\langle Y_{2}\right\rangle$ selecting only networks with a number $m$ of modules and we observed that this conditional average is a decreasing function of $m$, as figure 9 shows.

This behavior has a clear explanation in networks with $K=1$, where the modules are independent form the beginning. In this case, two configurations belong to the same attraction basin if and only if all their components on different modules belong to the attraction basins of the same local cycles. Since all such components are extracted independently, the probability of such an event is the product of the probabilities relative to individual modules and vanishes as the number of modules increases. This reasoning can not be applied to networks with connectivity larger than one, as we said previously, but the result that the average value of $Y_{2}$ decreases with the number of modules remains valid also in this case.

On the other hand, the average weight of the attraction basins has not a monotonic behavior as a function of the number of relevant elements in the network, $R$. This fact 


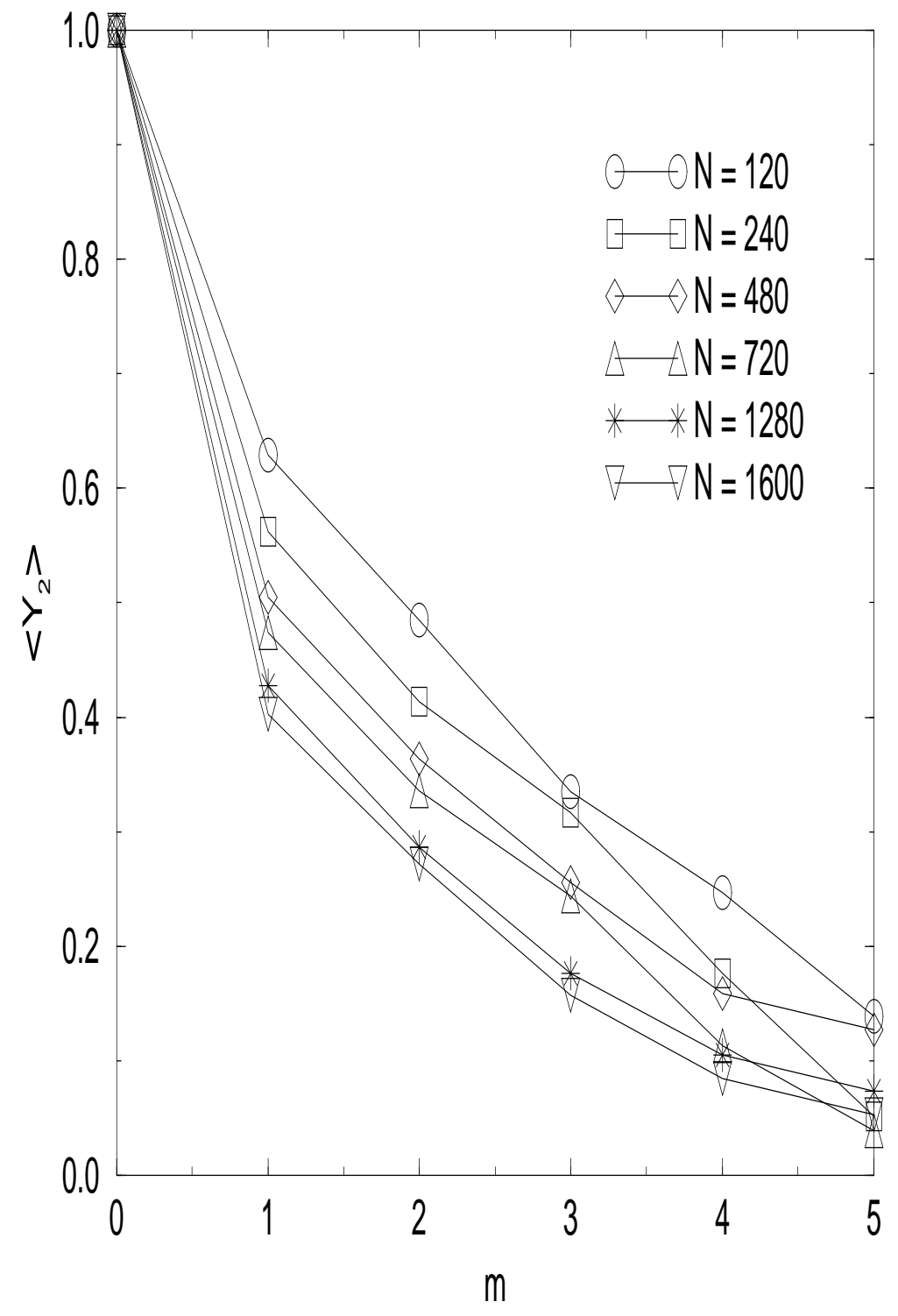

Figure 9: Conditional average of $Y_{2}=\sum_{\alpha} W_{a}^{2}$ in networks with $m$ modules. $K=4, \rho=1 / 4$. 
can be explained as a consequence of the fact that the number of modules does not grow monotonically with the number of relevant elements in the network. In the low-connectivity regime the number of modules increases with $R$, thus the weight of the attraction basins decreases. The contrary happens in the high-connectivity regime.

These characteristics do not change in the chaotic phase. Also for chaotic systems the average weight of the attraction basins decreases with the number of modules in the network, but in this case the probability to find more than one module goes to zero in the infinite size limit, and the effects of the modular organization disappear. In particular, the average basin weight remains finite and its limit value appears to be very close to the Random Map value $\left\langle Y_{2}\right\rangle=2 / 3$, as predicted by the annealed approximation [6].

We observed in simulations reported in [6] that in chaotic systems not far from the critical line $(K=3, \rho=1 / 2)$ the average basin weight, $\left\langle Y_{2}\right\rangle$, is not a monotonic function of system size: at first it decreases with $N$, then attains a minimum value of about 0.59 at $N=40$ and starts to increase, apparently toward the Random Map value of $2 / 3$. One could think that this not monotonic behavior is related to the modular structure, supposing that its effects are not monotonic in system size. This does not appear to be the case, as the same not monotonic behavior as a function of system size can be observed restricting the sample to networks with a single module (they are the big majority even for small sizes, in the chaotic phase). Nevertheless, it is possible that the extension of the concept of modules that we proposed in section 2 in order to fill the gap between the observed distribution of modules and the effects of factorization of cycle lengths can give a better comprehension of this behavior.

\section{Discussion}

The dynamical transition taking place in Kauffman networks can be characterized as a percolation transition in the set of the relevant elements (the analogy with percolation was already proposed by Derrida and Stauffer with respect to Random boolean networks on a lattice [5]).

In the frozen phase, the relevant elements are finite in number also in the infinite size limit and they are divided into asymptotically independent modules. In the chaotic phase all the relevant elements belong to the same cluster, and their number is proportional to system size. At the border between these two phases, the critical line is characterized by a number of relevant elements increasing as $\sqrt{N}$ and a number of modules that grows with system size (apparently with a logarithmic behavior).

Critical networks have a wide range of modular structures, ranging from networks reminiscent of the frozen phase (with few relevant elements and low effective connectivity and a number of modules that increases with the number of relevant elements) to networks reminiscent of the chaotic phase (with many relevant elements, larger effective connectivity and a number of modules that decreases with the number of relevant elements).

The modular organization can explain qualitatively - and, maybe, also quantitatively

- the main properties of the attractors of critical networks. These attractors are the composition of the undecomposable cycles of the elements of the single modules, so that their 
length is the minimal common multiple of the lengths of these cycles and their number is the product of the numbers of these cycles. One expects then that networks closer to the frozen phase, whose modules have effective connectivity very close to 1 , are characterized by a large number of attractors (exponentially increasing with $R$, the number of relevant elements) whose length increases as $R$ or faster. In networks close to the chaotic phase, on the other hand, one expects to find less attractors (for the same value of $R$ ), but longer ones. This is in agreement with the observed distributions of the number and of the length of the attractors, that become broader and broader as system size increases.

It seems to us that these results imply that the typical values of the number and of the length of the attractors increase asymptotically faster than any power law. In simulations of small systems, Kauffman [1] observed that this quantities behave as $\sqrt{N}$. Simulating larger critical systems [8], we observed that the distributions of these quantities are compatible with two effective scales, one increasing as $\sqrt{N}$ and another one, for rare but not vanishingly rare networks, increasing as a stretched exponential of $N$. This observation is consistent with the conclusions that can be drawn from the study of the modular organization.

The study of the modular structure can also explain why the average weight of the attraction basins of critical networks vanishes in the infinite size limit.

Thus it would be interesting to investigate analytically the distribution of size and number of the modules. We think that the approach that we used to estimate the number of relevant elements, consisting in adding a new element to the network and computing the modification of the modular structure, could be a good tool for a more quantitative understanding of the modular organization of critical networks, if the approximation that we used can be in some way controlled.

The modular organization of Kauffman networks is a consequence of the finite connectivity of this model. It is because of the finite connectivity that some elements become stable, and act as a barrier in the transmission of information among different modules. Thus the structures described in this work are peculiar to this kind of cellular automata, and they are not common to other models, for other aspects very similar to this one, such as asymmetric neural networks. It would be interesting to understand if and how the existence of a modular organization is related to the biological situation that Kauffman networks are intended to model, i.e. genetic regulatory systems.

We think that the spontaneous emergence of a modular organization in critical networks is one of the most interesting features of such systems, and could have played an important role in the emergence of cellular organization from an hypothetical network of chemical reactions where the primordial regulatory rules were of a probabilistic type. One of the advantages of modularity is the possibility to build many independent objects, which could have been shaped more easily by natural selection. This interpretation is highly speculative but very appealing, and raises for the biological modelization the challenging question about the mechanisms which could drive to criticality such an hypothetical ensemble of self regulated chemical reactions.

We argue from this study that for large system size the typical scale of the number and of the length of the attractors increase much faster than $\sqrt{N}$, as it was observed by Kauffman in early works [1]. The $\sqrt{N}$ law was considered by Kauffman an important argument in favor of the fact that randomly assembled networks of regulatory systems can behave in a 
way very reminiscent of the behavior of true biological organisms. This law, and thus the biological analogy, still holds approximately for a small enough number of elements. When the number of genes becomes large, on the other hand, the number of cycles (in the biological metaphor, the number of cellular types) and especially their length (which in the biological metaphor represents cycle cell time) become too large to allow a biological interpretation. In this case it seems unlikely that critical random boolean networks behave as control networks in the cells, and that the scaling laws observed for real organisms are just a reflex of the typical properties of random regulatory networks. Random boolean networks with a number of elements of the same order as the number of genes in higher eukariots have with finite probability too many and too long attractors, and some kind of selection must come into play to maintain the approximate power law scaling observed for small systems. But it may be that genetically interesting networks are not vanishingly rare in the ensemble of critical Kauffman networks in the infinite size limit. In the biological picture proposed by Kauffman, this would mean that some properties of real cells can still be interpreted as typical properties of an ensemble of randomly assembled regulatory networks, even if this ensemble has not to be identified with the whole ensemble of critical Kauffman networks.

\section{Acknowledgments}

U.B. would like to thank Henrik Flyvbjerg and Peter Grassberger for interesting discussions and for reading the manuscripts.

\section{References}

[1] S.A. Kauffman (1969), Metabolic Stability and Epigenesis in Random Constructed Genetic Nets, J. Theor. Biol. 22, 437-467.

[2] S.A. Kauffman (1990), Origins of Order: Self-Organization and Selection in Evolution, Oxford University Press

[3] U. Bastolla and G. Parisi (1996), Relevant Elements, Magnetization and Dynamical Properties in Kauffman Networks: a Numerical Study, Physica D.

[4] H. Flyvbjerg, N.J. Kjaer (1988), Exact Solution of Kauffman Model with Connectivity One, J.Phys. A:Math.Gen. 21(7), 1695-1718

[5] B. Derrida, D. Stauffer (1986): Phase Transitions in Two-dimensional Kauffman Cellular Automata, Europhys. Lett. 2(10), 739-745

[6] U. Bastolla and G. Parisi (1996), Closing Probabilities in the Kauffman model: an Annealed Computation, Physica D 98, 1

[7] H. Flyvbjerg (1988), An Order Parameter for Networks of Automata, J.Phys. A:Math.Gen. 21 L955-L960

[8] U. Bastolla and G. Parisi (1996), The Critical Line of Kauffman Networks, Journal of Theoretical Biology, in press. 
[9] B. Derrida and H. Flyvbjerg (1986), Multivalley Structure in Kauffman's Model: Analogy with Spin Glasses, J.Phys.A: Math.Gen. 19, L1003-L1008

[10] G. Paladin and A. Vulpiani (1987), Anomalous Scaling Laws in Multifractal Objects, Phys. Rep. 156, 147-225. 